\title{
Influence of Printhead Vibration in Impact Printer on Printing Quality*
}

\author{
Taichi SATO**, Kihachiro TANAKA**, \\ Yoshiharu MOURI** and Toshiyuki TAMATSU***
}

In order to obtain good printing quality with impact printers, a method of reducing vibration by carriage mounting printheads is investigated using a newly developed simulator for vibration analysis and experimental tests. As a result of a survey of various parameters, optimizing the carriage driving control pattern and increasing the stepper motor damping proved to be effective for decreasing carriage vibration. Consequently, significant improvement in printing quality is obtained.

Key Words: Vibration Control, Transient Response, Simulation, Mechatronics, Positioning, Business Equipment

\section{Introduction}

With the recent rapid demand for high-speed and high-quality printing from impact printers, improving the precision with which the printhead is positioned above the platen is more urgently required. Normally, the printhead shows increased vibration levels with increased speed. Therefore, conventional printhead driving conditions and driving parts encounter difficulties in satisfying this requirement.

The problems of dynamic behavior of printheads in driving directions have been studied by several researchers ${ }^{(1)(2)}$. However, it is not clear how printhead driving conditions and driving parts affect printhead vibration and printing quality.

For clarification, the authors developed a simulator for vibration analyses of carriage driving sys-

- Received 31st July, 1989

** Mechanical Engineering Research Laboratory, Hitachi, Ltd., 502 Kandatsu-machi, Tsuchiura, Ibaraki, 300, Japan

*** Mito Works, Hitachi, Ltd., 1070 Ichige, Katsuta, Ibaraki, 312, Japan tems. Using this simulator, the influence of driving conditions and driving parts on the dynamic behavior of carriage driving systems is investigated.

This paper describes a method by which printhead vibration in the driving direction, caused by the carriage driving system in an impact printer, is reduced by optimizing the carriage diving control pattern and increasing the carriage stepper motor damping.

\section{Simulator on Printhead Vibration in Driving Direction}

\subsection{Construction of an impact printer and its printing quality}

Figure 1 illustrates schematically the construction of the carriage driving system of the impact printer tested. The carriage driving system consists mainly of the stepper motor, the carriage-mounted printhead, and their connecting belt. When the stepper motor directly drives the belt that in turn drives the carriage back and forth across the paper, the carriage vibrates in the driving direction. In this vibrating condition, the printhead with 24 print wires impacts the paper on the platen through an ink ribbon. As the 
result of the printhead vibration, printing quality is decreased.

\section{2 Vibration model}

To clarify the vibration characteristics of the carriage driving system, a simulator for vibration analysis is developed. This vibration model can be approximated by the simulator with four degrees of freedom, as shown in Fig. 2 ; therefore, the simulator solves the fourth-order simultaneous differential equation using the Runge-Kutta-Gill method. The system consists of four mass elements which are the sum mass of both carriage and printhead, $m$; the mass of stepper motor, $M$; and the two directional moments of inertia of the stepper motor, $I$ and $J$; and four spring elemets, stiffnesses of belts $k_{1}, k_{2}$, and $k_{3}$, the electromagnetic torsional spring of stepper motor $k_{\varphi}$, the stepper motor's supporting stiffnesses, $k_{x}$ and

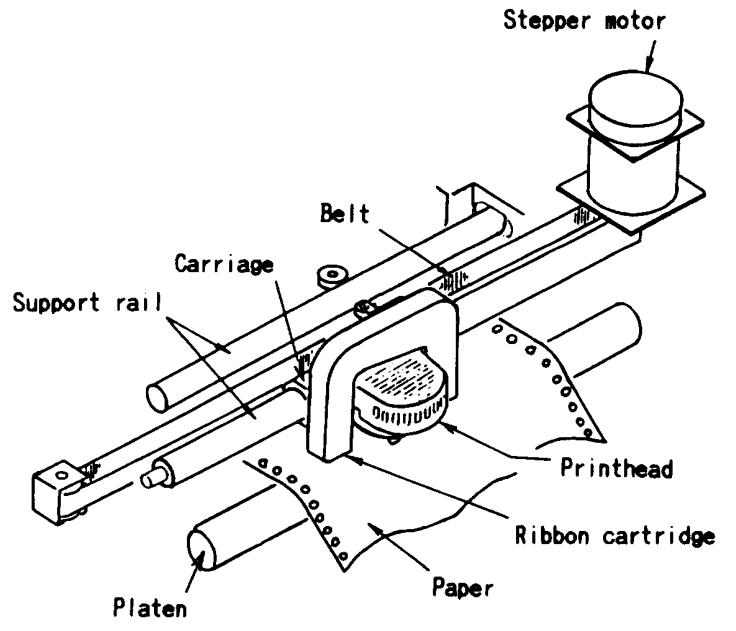

Fig. 1 Schematic illustration of impact printer

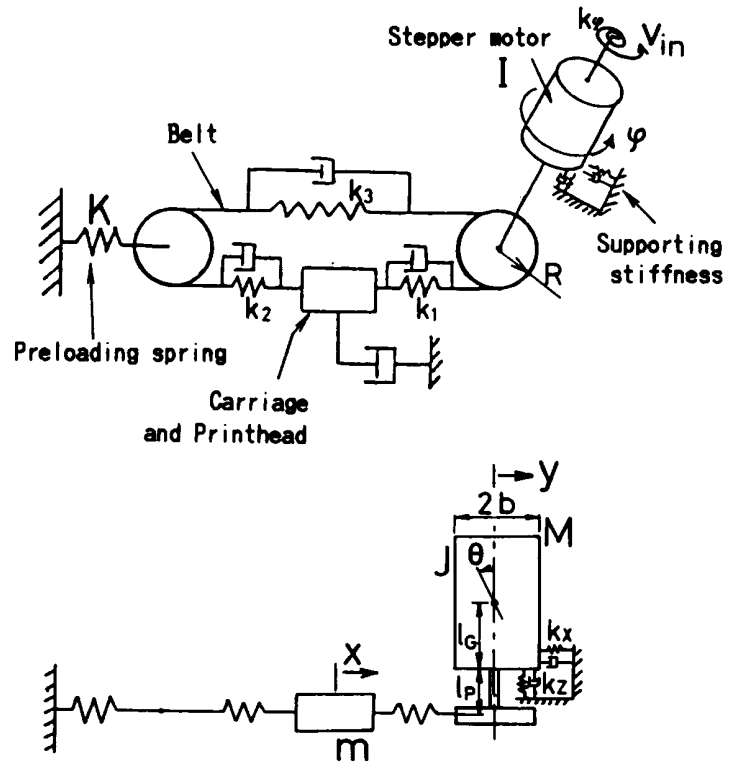

Fig. 2 Vibration model $k_{z}$, and preloading spring $K$, and their damping elements. These elements are determined by measurements and experimental tests. The carriage stepper motor's rotational velocities, $V_{i n}$, are put into the simulator as the driving condition. The equation of the system without damping is written as follows.

$$
\begin{aligned}
& {\left[\begin{array}{llll}
m & 0 & 0 & 0 \\
0 & M & 0 & 0 \\
0 & 0 & J & 0 \\
0 & 0 & 0 & I
\end{array}\right]\left[\begin{array}{l}
\ddot{x} \\
\ddot{y} \\
\ddot{\theta} \\
\ddot{\phi}
\end{array}\right]+\left[\begin{array}{ll}
k_{1}+K^{\prime} & -k_{1}+K^{\prime} \\
-k_{1}+K^{\prime} & k_{1}+K^{\prime}+k_{x} \\
& \\
l\left(-k_{1}+K^{\prime}\right) & l\left(k_{1}+K^{\prime}\right) \\
& +k_{x} l_{G} \\
-R\left(k_{1}+K^{\prime}\right) & R\left(k_{1}-K^{\prime}\right)
\end{array} *\right.} \\
& \left.\begin{array}{ll}
l\left(-k_{1}+K^{\prime}\right) & -R\left(k_{1}+K^{\prime}\right) \\
l\left(k_{1}+K^{\prime}\right) & R\left(k_{1}-K^{\prime}\right) \\
+k_{x} l_{c} & \\
l^{2}\left(k_{1}+K^{\prime}\right) & l R\left(k_{1}-K^{\prime}\right) \\
+k_{x} l_{G}^{2}+k_{z} b^{2} \\
l R\left(k_{1}-K^{\prime}\right) & R^{2}\left(k_{1}+K^{\prime}\right)+k_{\varphi}
\end{array}\right]\left\{\begin{array}{c}
x \\
y \\
\theta \\
\phi
\end{array}\right\}=\left\{\begin{array}{c}
0 \\
0 \\
0 \\
k_{\varphi} \phi_{i}
\end{array}\right\}
\end{aligned}
$$

where

$$
\begin{aligned}
& K^{\prime}=1 /\left(\frac{k_{2}+k_{3}}{k_{2} k_{3}}+\frac{2}{K}\right) \\
& l=l_{C}+l_{P} \\
& \phi_{i}=\frac{V_{i n}}{R} t \quad(t ; \text { time })
\end{aligned}
$$

Differential equation ( 1 ) is solved considering each part's damping.

Figure 3 shows simulation results for the time history response. Here, each wave, from top to dottom, is presented as follows.

1) Carriage driving control pattern (velocity) $\mathrm{V}$

2) Carriage theoretical displacement Disp.

3) Loaded torque on the stepper motor Torq.

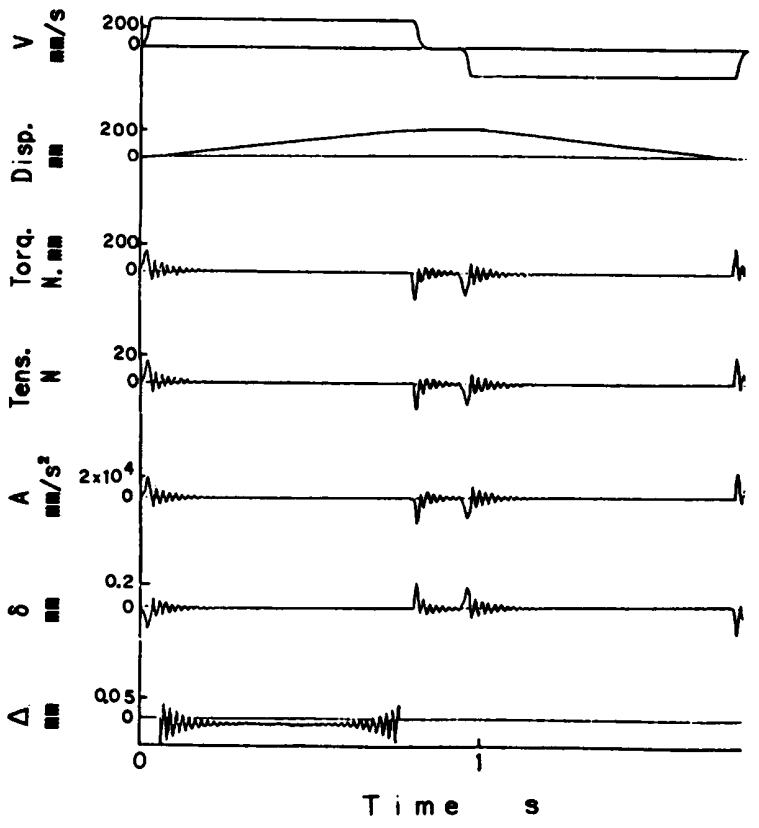

Fig. 3 Simulation results

JSME International Journal 
4) Fluctuating tension of preloading spring Tens.

5) Acceleration of carriage in driving direction $A$

6) Delay of carriage from the Disp. $\delta$

7) Difference of $\delta$ between backward and forward directions in the printing range $\Delta$.

\section{3 Accuracy of the simulator}

Printhead vibration is measured by the accelerometer mounted at the side of the printhead in the driving direction as the test impact printer is operating.

Figure 4 shows the simulation result and the experimental result of printhead acceleration.

It is clarified that the simulation result agrees with the experimental result for both acceleration level and natural period $T_{n}$.

\section{Optimizing Carriage Driving Pattern}

\section{1 Influence of accelerating time}

The influence of accelerating time, $t_{0}$, is investigated in experimental tests, as shown in Fig. 5, with the settling time when the printhead acceleration in the driving direction shows $2 \times 10^{4} \mathrm{~mm} / \mathrm{s}^{2}$. The filled in symbols show the results in the case of forward direction driving; the open-point symbols are the opposite direction results. When $t_{0}$ is in the vicinity of $T_{n}$, the settling time is shortest. Therefore, at this accelerating time $t_{0}\left(=T_{n}\right)$, the carriage driving system performs well. This result is appropriate, without any detailed simulation, considering the characteristics that the shock spectrum, for the base excited vibratory system with one degree of freedom, shows a minimum value at $t_{0}=T_{n}$.

A printing result in the case of $t_{0}=T_{n}$ is compared with the result for $t_{0}=0.7 T_{n}$, as shown in Fig. 6 . When

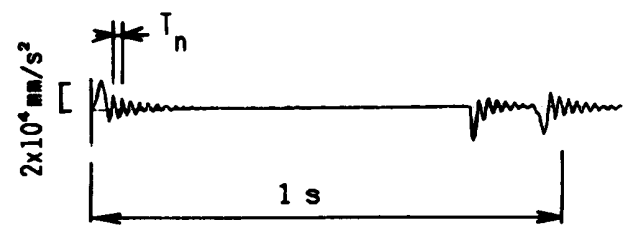

(a) Simulation

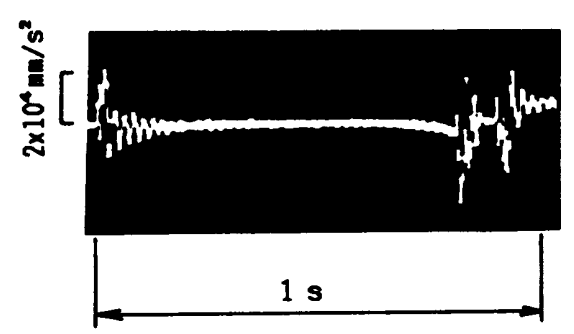

(b) Experiment

Fig. 4 Acceleration of printhead-Verification of the simulator $t_{0}$ is not eqaul to $T_{n}$, the vertical line of symbol $\bigotimes$ is distorted. However, in the case of $t_{0}=T_{n}$, its line becomes straight.

Consequently, good printing results from decreased printhead vibration in both driving directions.

\subsection{Influence of driving control pattern shape}

Formerly, when $t_{0}$ was not equal to $T_{n}$, the printhead vibrated. Therefore, in the case of $t_{0} \neq T_{n}$, it was necessary that the carriage driving control pattern shape (called the velocity pattern) was improved to obtain good vibratory characteristics (printing quality).

The authors investigated the velocity pattern $-\mathbf{a}$ trapezoidal velocity pulse added to the trapezoidal velocity pattern (as shown in Figs. 7 and 8). In this paper, this pattern is called a "double trapezoidal pattern".

Figure 7 shows the peak-to-peak value of position difference between the backward and forward directions (called position difference), that is, $\Delta_{\max }{ }^{-}$ $\Delta_{\min }$ versus the bottom time of trapezoidal velocity pulse $T_{0}$. A minimum position difference occurs in the vicinity of $T_{0} / T_{n}=1.0$ and 2.0. Figure 8 shows both the simulation results and the experimental results on printhead acceleration when $T_{0}$ equals $T_{n}$.

\subsection{Characteristics of double trapezoidal pat-} terns

This paragraph discusses in detail the effects of

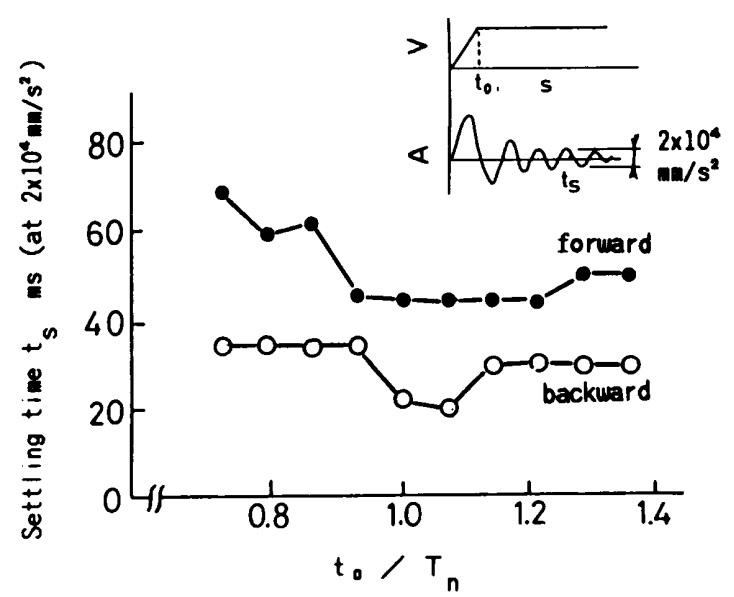

Fig. 5 Settling time of printhead acceleration $t_{s}$ vs accelerating time $t_{0}$
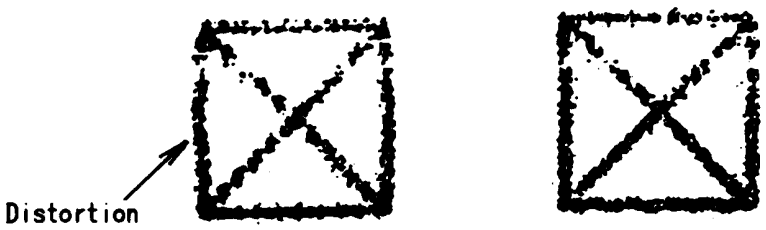

$t_{0} / T_{n}=0.7$

$t_{0} / T_{n}=1.0$

Fig. 6 Printing results 
double trapezoidal paterns on vibratory characteristics. Here, the vibratory system with one degree of freedom excited at the base is considered.

The differential equation of the system with mass, $m$, and spring, $k$, is written as follows.

$$
\begin{aligned}
& \ddot{\nu}(t) / \omega_{n}{ }^{2}+\nu(t)=\xi(t) \\
& \omega_{n}{ }^{2}=k / m
\end{aligned}
$$

Here $\xi(t)$ is the input velocity on the base and $\nu(t)$ is the output velocity of the mass. The solution $\nu(t)$ is as follows.

$$
\nu(t)=\int_{0}^{t} \xi(\tau) h(t-\tau) d \tau
$$

where

$$
h(t)=\omega_{n} \sin \omega_{n} t
$$

If the double trapezoidal pattern is defined as shown in

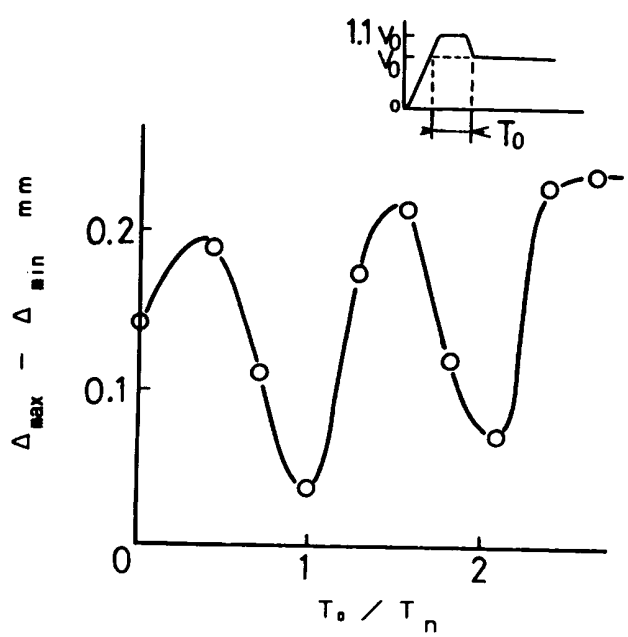

Fig. 7 Variation of $\Delta_{\max }-\Delta_{\min }$ with the time $T_{0}$ (simulation)

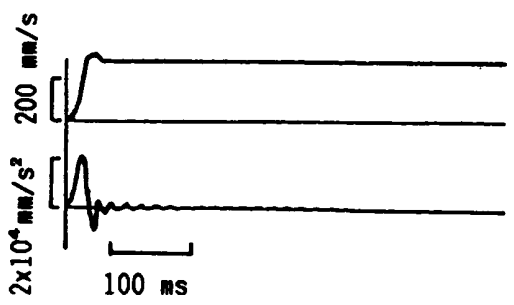

(a) Simulation

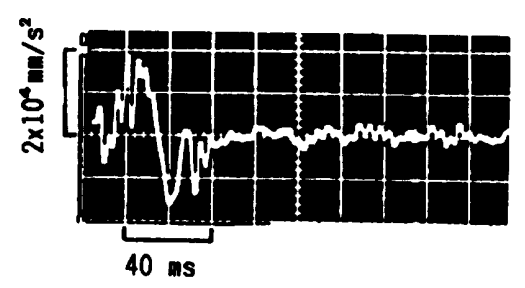

(b) Experiment

Fig. 8 Effect of double trapezoidal pattern on printhead vibration
Fig. 9, $\nu(t)$ is calculated as follows.

$$
\begin{aligned}
& \nu(t)=p \xi_{c}\left(\frac{t}{t_{1}}-\frac{\sin \omega_{n} t}{\omega_{n} t_{1}}\right) \quad\left(0 \leqq t \leqq t_{1}\right) \\
& \nu(t)=p \xi_{c}\left\{1+\frac{\sin \omega_{n}\left(t-t_{1}\right)-\sin \omega_{n} t}{\omega_{n} t_{1}}\right\} \quad\left(t_{1} \leqq t<t_{2}\right) \\
& \nu(t)=p \xi_{c}\left\{1-\frac{t-t_{2}}{t_{1}} \quad(6) \quad\left(t_{2} \leqq t<t_{3}\right)\right. \\
& \left.-\frac{\sin \omega_{n} t-\sin \omega_{n}\left(t-t_{1}\right)-\sin \omega_{n}\left(t-t_{2}\right)}{\omega_{n} t_{1}}\right\} \\
& \nu(t)=p \xi_{c}\left\{\frac{1}{p}+\left(1-\frac{1}{p}-\frac{t_{3}-t_{2}}{t_{1}}\right) \cos \omega_{n}\left(t-t_{3}\right)\right.
\end{aligned}
$$

$\left.-\frac{\sin \omega_{n} t-\sin \omega_{n}\left(t-t_{1}\right)-\sin \omega_{n}\left(t-t_{2}\right)+\sin \omega_{n}\left(t-t_{3}\right)}{\omega_{n} t_{1}}\right\}$

where

$$
\begin{aligned}
& t_{1}=p t_{0} \\
& t_{2}=(2-p) t_{0}+T_{0} \\
& t_{3}=t_{0}+T_{0}
\end{aligned}
$$

The value, $\nu(t)$, in the time range, $t_{3} \leqq t$, appears to be dynamically significant, and thus its maximum value is given as follows.

$$
\begin{gathered}
\nu(t)_{\max }=p \xi_{c}\left[\frac{1}{p}+\sqrt{\left\{\left(1-\frac{1}{p}-\frac{t_{3}-t_{2}}{t_{1}}\right) \cos \frac{2 \pi t_{3}}{T_{n}}\right.}\right. \\
\frac{\left.-\frac{T_{n}\left(\sin \frac{2 \pi t_{1}}{T_{n}}+\sin \frac{2 \pi t_{2}}{T_{n}}-\sin \frac{2 \pi t_{3}}{T_{n}}\right)}{2 \pi t_{1}}\right\}^{2}}{+\left\{\left(1-\frac{1}{p}-\frac{t_{3}-t_{2}}{t_{1}}\right) \sin \frac{2 \pi t_{3}}{T_{n}}\right.} \\
\left.\left.-\frac{T_{n}\left(1-\cos \frac{2 \pi t_{1}}{T_{n}}-\cos \frac{2 \pi t_{2}}{T_{n}}+\cos \frac{2 \pi t_{3}}{T_{n}}\right)}{2 \pi t_{1}}\right\}^{2}\right]
\end{gathered}
$$

$T_{n}=2 \pi / \omega_{n}$

Figure 10 shows the residual shock spectrum, $\nu(t)_{\max } / \xi_{c}$ vs $T_{0} / T_{n}$, in the case of $t_{0}=10 \mathrm{~ms}$ and $t_{0}=30$ ms. By choosing the proper $p$ value, the minimum values of $\nu(t)_{\max }$ are observed at $T_{0}=T_{n}$. Therefore, in this case, the vibratory system performs well.

\section{Effect of Stepper Motor Damping}

The influence of stepper motor damping on printhead vibration is investigated as shown in Fig. 11 with the settling time when the printhead acceleration

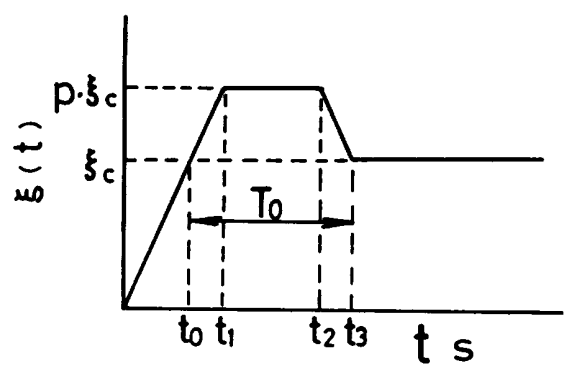

Fig. 9 Schematic diagram of double trapezoidal pattern 


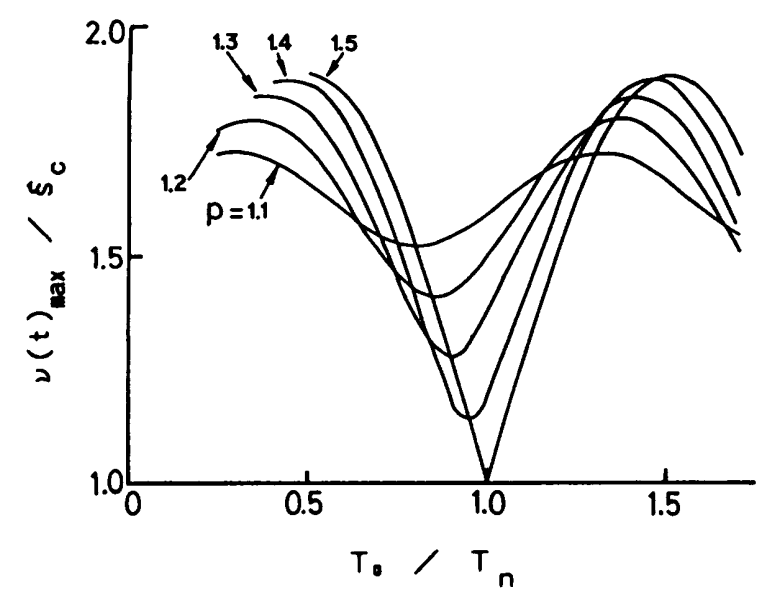

(a) $t_{0}=10 \mathrm{~ms}$

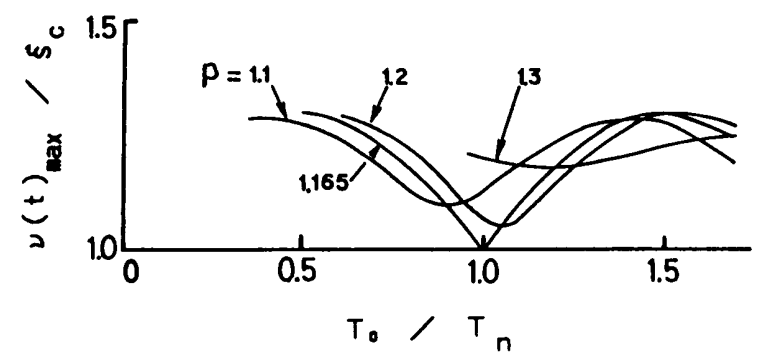

(b) $t_{0}=30 \mathrm{~ms}$

Fig. 10 Variations of $\nu(t)_{\max }$ with $T_{0}$

shows $1 \times 10^{4} \mathrm{~mm} / \mathrm{s}^{2}$. The solid line shows the simulation result and the open-point symbols are experimental results. As the damping factor of stepper motor $\zeta$ increases, the settling time, $t_{s}$, decreases.

Consequently, the use of a damper, fixed to the stepper motor, affords significant improvement.

\section{Conclusions}

The authors developed a simulator for vibration analyses of carriage driving systems, output printhead acceleration in driving directions, delay of carriage, difference of delay between backward and forward directions, and so on.

Using the simulator, the effects of driving condi-

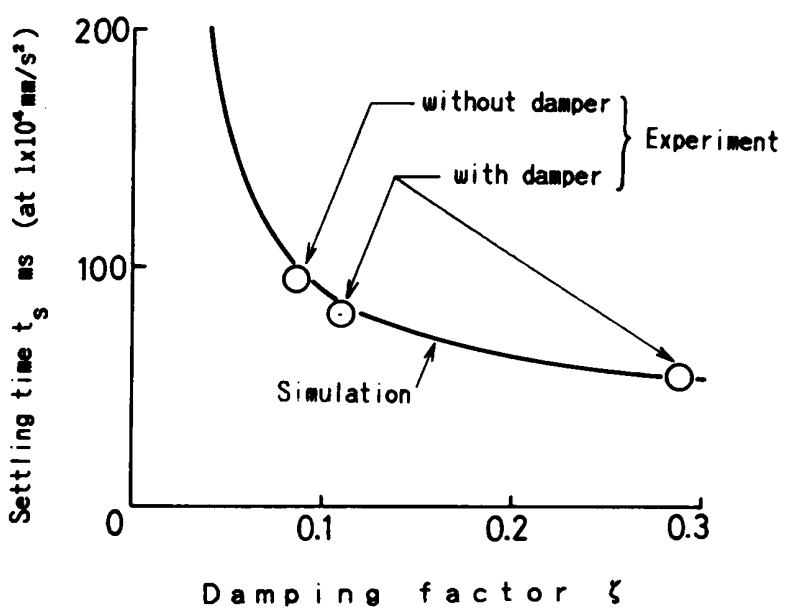

Fig. 11 Effect of damper, fixed to the stepper motor, on printhead vibration

tions and driving parts on the carriage driving systems are calculated. The following points have been clarified;

1. When the accelerating time is equal to the natural period of the carriage driving system, the system shows good vibratory characteristics and good printing quality.

2. In the double trapezoidal velocity pattern, as shown in Figs. 7 and 8, both the proper height and proper bottom time (that is the natural period) provide significant improvement in the vibratory characteristics of the carriage driving system.

3. By the use of a damper fixed to the stepper motor, printhead vibration in the driving direction is reduced.

\section{References}

(1) Nishimura, K., The Dynamics of Impact Printing in Belt Type Printer, Trans. Jpn. Soc. Mech. Eng., (in Japanese), Vol. 42, No. 353 (1976), p. 153.

(2) Iwasawa, N., Hashimoto, M. and Tagawa, N., Prepr. of Jpn. Soc. Mech. Eng., (in Japanese), No. 880-5(1988), p. 33. 